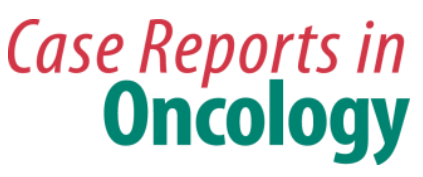

Case Rep Oncol 2017;10:840-845

DOI: $10.1159 / 000480452$

Published online: September 20, 2017

This article is licensed under the Creative Commons Attribution-NonCommercial 4.0 International License (CC BY-NC) (http://www.karger.com/Services/OpenAccessLicense). Usage and distribution for commercial purposes requires written permission.

\title{
Treatment of Leptomeningeal Metastases in a Patient with Non-Small Cell Lung Cancer Harboring EGFR T790M Mutation
}

\author{
Hardy Niu ${ }^{a}$ Junle Zhou ${ }^{b}$ Harvinder Maan ${ }^{c}$ Maurie Markman ${ }^{d}$ \\ Jiaxin $\mathrm{Niu}^{\mathrm{e}}$ \\ ${ }^{a}$ Basis Scottsdale, Banner MD Anderson Cancer Center, Gilbert, AZ, USA; ${ }^{b}$ Department of \\ Pharmacy, Banner MD Anderson Cancer Center, Gilbert, AZ, USA; ${ }^{c}$ Columbus Radiology \\ Corporation, Columbus, OH, USA; ${ }^{d}$ Department of Medical Oncology, Cancer Treatment \\ Centers of America, and Drexel University College of Medicine, Philadelphia, PA, USA; \\ ${ }^{e}$ Department of Medical Oncology, Banner MD Anderson Cancer Center, Gilbert, AZ, USA
}

\section{Keywords}

Lung cancer · Leptomeningeal metastasis · EGFR mutation · T790M · Liquid biopsy

\begin{abstract}
Background: Leptomeningeal metastasis (LM) is an uncommon complication in patients with solid tumors, associated with poor survival. However, LM appears to be more frequent in lung cancer patients with EGFR mutations, posing a unique clinical challenge to treating physicians. Case Presentation: We report the case of a 68-year-old Asian man with metastatic lung adenocarcinoma harboring an EGFR L858R mutation, which was initially treated with gefitinib. He developed disease progression 1 year later. Re-biopsy of the right lower lobe primary lesion revealed only an EGFR L858R mutation in the absence of a T790M mutation. The patient also experienced persistent confusion and generalized fatigue, and magnetic resonance imaging (MRI) of the brain demonstrated extensive LM. At this time, a liquid biopsy revealed an EGFR T790M mutation. Following initiation of treatment with osimertinib, the patient exhibited a rapid response with MRI of the brain showing substantial improvement of the LM after 6 months. Unfortunately, the LM recurred after 1 year at which time the patient declined further systemic chemotherapy. Conclusion: To our knowledge, this is the second
\end{abstract}




\section{Case Reports in Oncology}

Niu et al: Treatment of Leptomeningeal Metastases in a Patient with Non-Small Cell Lung Cancer Harboring EGFR T790M Mutation

reported case of LM in a patient with lung cancer harboring an EGFR T790M mutation that was successfully treated with osimertinib.

(C) 2017 The Author(s)

Published by S. Karger AG, Basel

\section{Introduction}

Metastatic non-small cell lung cancer (NSCLC) harboring epidermal growth factor receptor (EGFR) is often sensitive to treatment with EGFR tyrosine kinase inhibitors (TKIs). However, acquired resistance to TKIs is a prevalent clinical problem which typically occurs after a median of 10-12 months. It is most commonly mediated by T790M mutation. Recently, third-generation TKI osimertinib has been approved for the treatment of metastatic NSCLC with acquired T790M mutation.

Leptomeningeal metastasis (LM) is an uncommon complication of NSCLC with a dismal diagnosis. The role of TKIs in this setting is currently under clinical investigation. We report the case of a 68-year-old man with metastatic lung adenocarcinoma harboring EGFR mutation who presented with LM 1 year after initial response to gefitinib. Osimertinib was administered after T790M mutation was identified via liquid biopsy and the patient achieved a clinically meaningful response lasting approximately 1 year.

\section{Case Report}

A 68-year-old Asian man with no smoking history was diagnosed with right lower lobe lung adenocarcinoma with multiple osseous metastases but without brain metastasis in October 2014 when he presented with severe back pain. Molecular study of a tissue biopsy revealed EGFR mutation L858R, sensitive to EGFR TKIs. Treatment with gefitinib, a firstgeneration TKI, was initiated in November 2014. The patient tolerated therapy well and exhibited an excellent clinical response until November 2015 when he started to experience progressive worsening of lower back pain and bilateral lower extremity weakness. Imaging studies demonstrated disease progression, and thus re-biopsy of the right lower lobe lesion was performed to search for a T790M mutation, the most common molecular abnormality responsible for resistance to targeted therapy. Somewhat surprisingly, biopsy sample mutation studies again demonstrated EGFR L858R but without a T790M mutation. The patient declined systemic cytotoxic chemotherapy and palliative radiation to the lumbar spine metastasis, but continued with gefitinib.

The patient transferred his care to our center at the end of January 2016. Unfortunately, he continued to deteriorate clinically and developed intractable lower back pain and bilateral lower extremity weakness, but no incontinence. Repeat magnetic resonance imaging (MRI) of the spine showed metastases at multiple levels involving the thoracic, lumbar, and sacral spines without compromise of the central canal or compression of the spinal nerve roots. The patient received urgent palliative radiation of 35 Gy in 14 fractions from T7-S2 spine, demonstrating a favorable symptomatic response. The patient was subsequently hospitalized for persistent confusion, generalized fatigue, anorexia, and failure to thrive. Neurological examination revealed mental status changes, but no focal neurological deficits. MRI of the brain demonstrated extensive leptomeningeal carcinomatosis (Fig. 1a), which was believed to be the cause of his symptomatology. In view of the previous failed attempt to identify a T790M mutation via tissue biopsy, a liquid biopsy was undertaken which revealed an 


\section{Case Reports in Oncology}

EGFR T790M mutation. Osimertinib 80 mg p.o. daily was initiated despite lack of solid clinical evidence for the utility of this agent in LM.

The patient responded rapidly, displayed substantial clinical improvement within 2 weeks of starting therapy, and became symptom-free after 3 months of treatment. Six months after initiation of osimertinib, repeat MRI of the brain revealed marked reduction of LM (Fig. 1b). Unfortunately, the patient developed hearing loss, confusion, and short-term memory loss approximately 1 year later in February 2017. Computerized tomography of the abdomen showed a few enlarged retroperitoneal lymph nodes, consistent with metastasis. MRI of the brain demonstrated progression of LM involving the cerebellar vermis, right occipital lobe as well as bilateral temporal and parietal lobes. The patient declined further systemic chemotherapy and expired after a short time interval.

\section{Discussion}

LM has been reported to develop in approximately $1-5 \%$ of patients with metastatic solid tumors [1, 2]. Breast cancer, lung cancer, and melanoma are the 3 cancer types where LM is most commonly observed [1]. Unlike small cell lung cancer, the incidence of LM is approximately $1 \%$ for NSCLC with a dismal median survival of only 3 months after the diagnosis [2-4].

Interestingly, the incidence of LM has been reported to be higher (9.4\%) in NSCLC with EGFR mutation as compared with EGFR wild-type (1.7\%) in the largest reported series of over 5,000 patients [5]. The exact mechanism underlying this clinical phenomenon is unclear but it has been attributed to longer survival and poor penetration of the blood-brain barrier (BBB) by early-generation TKIs [5]. With wide application of TKIs, the overall survival in metastatic NSCLC patients with EGFR mutations has been prolonged significantly to up to 3 years, whereas the overall survival for lung adenocarcinoma was approximately 18 months before the introduction of immunotherapy [6,7]. The improved survival might allow lung cancer cells to cross the BBB via constant seeding and metastasize to the central nervous system (CNS).

Although initial gefitinib or erlotinib treatment was associated with a lower cumulative risk of CNS progression (6\%) compared with chemotherapy (19\%) at 12 months, it is also known that early TKIs have poor capability to penetrate the BBB [8]. In preclinical studies, the ratio of cerebral spinal fluid to plasma concentration for the first-generation (gefitinib, erlotinib) and second-generation (afatinib) EGFR inhibitors was approximately 1\% [9-11]. Thus, the brain may become a sanctuary site where the BBB may offer protection from early TKIs, resulting in increased CNS metastasis including LM over time.

In the AURA study, osimertinib exhibited a better response rate (61 vs. $21 \%$ ) and median progression-free survival (9.6 vs. 2.8 months) in patients with T790M-positive versus T790M-negative tumor upon progression on previous TKI therapy [12]. Therefore, the very first clinical question to answer in this case was whether this patient had acquired a T790M mutation. The attempt with tissue biopsy outside our facility failed to reveal this mutation. Liquid biopsy on cell-free circulating tumor DNA has recently been under intense clinical studies. In the AURA study, of 58 patients with T790M-negative tumors, T790M was detected in plasma of 18 (31\%). More importantly, objective response rate and median progression-free survival were similar in patients with T790M-positive tumor results, indicating the complementary role of plasma testing to tissue testing in an EGFR TKI-resistant setting [13]. 


\section{Case Reports in Oncology}

\begin{tabular}{l|l}
\hline DOI: $10.1159 / 000480452$ & (c) 2017 The Author(s). Published by S. Karger AG, Basel
\end{tabular} www.karger.com/cro

Niu et al.: Treatment of Leptomeningeal Metastases in a Patient with Non-Small Cell Lung Cancer Harboring EGFR T790M Mutation

Taking advantage of plasma-based liquid biopsy, we were able to successfully identify a T790M mutation without requiring further biopsy.

It is challenging to treat patients with NSCLC who have developed LM. There are no prospective studies or official guidelines in regard to how to manage LM. Traditionally, wholebrain radiation therapy has been regarded as the cornerstone of treatment. Its clinical benefits, however, have been called into question by a large phase III trial (QUARTZ study) and in particular in patients with a poor performance status such as our case.

Previously, gefitinib was shown to benefit EGFR-mutant NSCLC patients with a poor performance status [14]. In contrast to the early TKIs, the third-generation TKI osimertinib, recently approved to treat lung cancer harboring a T790M mutation after progression on another EGFR inhibitor, exhibited impressive CNS penetration. The concentration in the brain was 5- to-25-fold higher than plasma in a mouse model [15]. Moreover, treatment with osimertinib led to regression of LM refractory to the early TKIs in a LM mouse model [16].

At the time of initiation of treatment in the patient presented here, there were no reported cases available to address this challenging scenario. Given the patient's poor performance status (ECOG 4) and preclinical activity of osimertinib for LM, it was felt to be reasonable to attempt osimertinib at that time. The patient achieved a rapid and durable response for 1 year before he developed progression of LM. Repeat liquid biopsy revealed no T790M mutation, but only a neurofibromin (NF1) W267R mutation. The functional consequences of this mutation were unknown at this point, but conceivably involved in the resistance to osimertinib therapy.

While the patient was receiving therapy, the promising results of an early phase I study were presented using osimertinib to treat LM in patients with an EGFR mutation without previous TKI therapy [17]. Another case reported was also recently published with a very similar result to the present case. Taken together, it appears that osimertinib can be used in the frontline or second-line setting in LM [18].

\section{Conclusion}

In summary, to the best of our knowledge, this is the second reported case of LM in a patient with lung cancer harboring an EGFR T790M mutation that was successfully treated with osimertinib. Further prospective studies are needed in this challenging clinical setting.

\section{Statement of Ethics}

This is a retrospective case report and IRB approval was not needed.

\section{Disclosure Statement}

The authors have nothing to disclose. 


\section{Case Reports in Oncology}

\begin{tabular}{l|l} 
Case Rep Oncol 2017;10:840-845 \\
\hline DOI: 10.1159/000480452 & $\begin{array}{l}\text { (c) 2017 The Author(s). Published by S. Karger AG, Basel } \\
\text { www.karger.com/cro }\end{array}$ \\
\hline
\end{tabular}

Niu et al.: Treatment of Leptomeningeal Metastases in a Patient with Non-Small Cell Lung Cancer Harboring EGFR T790M Mutation

\section{References}

1 Le Rhun E, Taillibert S, Chamberlain MC: Carcinomatous meningitis: leptomeningeal metastases in solid tumors. Surg Neurol Int 2013;4(suppl 4):S265-S288.

-2 Lee SJ, et al: Leptomeningeal carcinomatosis in non-small-cell lung cancer patients: impact on survival and correlated prognostic factors. J Thorac Oncol 2013;8:185-191.

-3 Riess JW, et al: Prolonged survival of patients with non-small-cell lung cancer with leptomeningeal carcinomatosis in the modern treatment era. Clin Lung Cancer 2014;15:202-206.

-4 Park JH, et al: Clinical outcomes of leptomeningeal metastasis in patients with non-small cell lung cancer in the modern chemotherapy era. Lung Cancer 2012;76:387-392.

5 Li YS, et al: Leptomeningeal metastases in patients with NSCLC with EGFR mutations. J Thorac Oncol 2016;11:1962-1969.

-6 Lin JJ, et al: Five-year survival in EGFR-mutant metastatic lung adenocarcinoma treated with EGFRTKIs. J Thorac Oncol 2016;11:556-565.

-7 Yang JC, et al: Afatinib versus cisplatin-based chemotherapy for EGFR mutation-positive lung adenocarcinoma (LUX-Lung 3 and LUX-Lung 6): analysis of overall survival data from two randomised, phase 3 trials. Lancet Oncol 2015;16:141-151.

-8 Heon S, et al: The impact of initial gefitinib or erlotinib versus chemotherapy on central nervous system progression in advanced non-small cell lung cancer with EGFR mutations. Clin Cancer Res 2012;18:4406-4414.

9 Deng Y, et al: The concentration of erlotinib in the cerebrospinal fluid of patients with brain metastasis from non-small-cell lung cancer. Mol Clin Oncol 2014;2:116-120.

10 Togashi Y, et al: Cerebrospinal fluid concentration of gefitinib and erlotinib in patients with non-small cell lung cancer. Cancer Chemother Pharmacol 2012;70:399-405.

11 Hoffknecht P, et al: Efficacy of the irreversible ErbB family blocker afatinib in epidermal growth factor receptor (EGFR) tyrosine kinase inhibitor (TKI)-pretreated non-small-cell lung cancer patients with brain metastases or leptomeningeal disease. J Thorac Oncol 2015;10:156-163.

12 Janne PA, et al: AZD9291 in EGFR inhibitor-resistant non-small-cell lung cancer. N Engl J Med 2015;372:1689-1699.

13 Oxnard GR, et al: Association between plasma genotyping and outcomes of treatment with osimertinib (AZD9291) in advanced non-small-cell lung cancer. J Clin Oncol 2016;34:3375-3382.

$\checkmark 14$ Inoue A, et al: First-line gefitinib for patients with advanced non-small-cell lung cancer harboring epidermal growth factor receptor mutations without indication for chemotherapy. J Clin Oncol 2009;27:1394-1400.

15 Ballard P, et al: Preclinical comparison of osimertinib with other EGFR-TKIs in EGFR-mutant NSCLC brain metastases models, and early evidence of clinical brain metastases activity. Clin Cancer Res 2016;22:5130-5140.

16 Nanjo S, et al: High efficacy of third generation EGFR inhibitor AZD9291 in a leptomeningeal carcinomatosis model with EGFR-mutant lung cancer cells. Oncotarget 2016;7:3847-3856.

17 Yang JC, Kim DW, Kim SW, et al: Osimertinib activity in patients with leptomeningeal disease from nonsmall cell lung cancer: updated results from the BLOOM study. J Clin Oncol 2016;34(15 suppl):abs 9002.

18 Sakai H, Hayashi H, Iwasa T, et al: Successful osimertinib treatment for leptomeningeal carcinomatosis from lung adenocarcinoma with the T790M mutation of EGFR. ESMO Open 2017;1:e000104 


\section{Case Reports in Oncology}
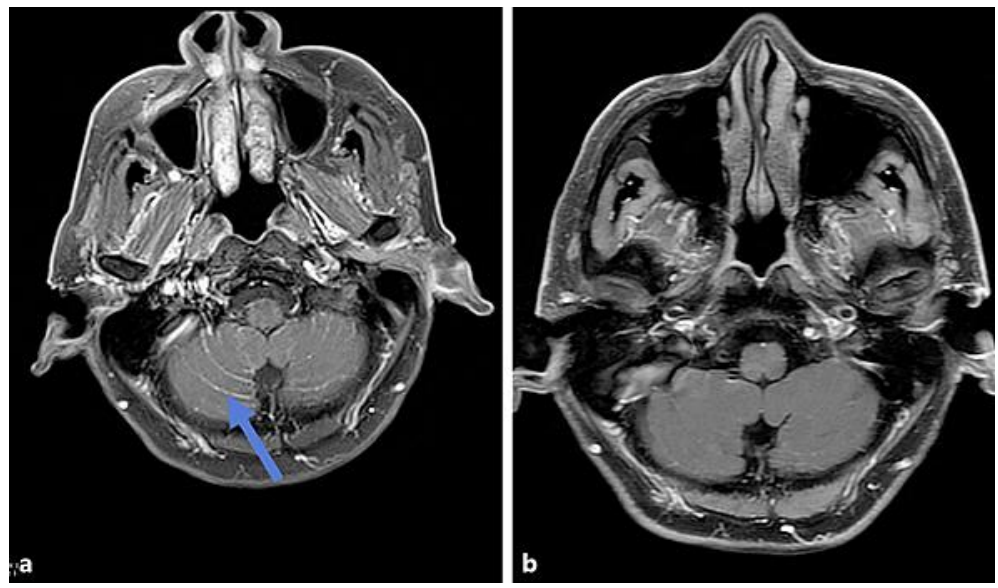

Fig. 1. a Brain MRI (2/2/2016): post-contrast T1 fat suppressed images demonstrating extensive leptomeningeal carcinomatosis throughout the cerebellar folia in a pattern classically described as "Zuckerguss" or sugar icing (arrow). b Brain MRI (10/31/2016): post-contrast T1 fat suppressed images after the administration of osimertinib demonstrating marked reduction of leptomeningeal enhancement of the cerebellar folia consistent with treatment response. 